\title{
Primacy and frequency effects in absolute judgments of visual velocity
}

\author{
ALEXANDER SOKOLOV \\ University of Tübingen, Tübingen, Germany \\ MARINA PAVLOVA \\ Max Planck Institute for Biological Cybernetics, Tübingen, Germany \\ and \\ WALTER H. EHRENSTEIN \\ Institut für Arbeitsphysiologie, Dortmund, Germany
}

\begin{abstract}
In absolute judgment tasks, identical stimuli are rated higher (or lower) when presented in a series of more frequent small (or large) stimuli. Using visual stimuli differing in velocity, we show that this conventional frequency effect is largely modulated by the primacy effect-that is, by the stimuli occurring on the early trials of a run. In Experiment 1, a frequency-like primacy effect was obtained with equal-frequent velocities. Identical velocities were rated faster when mainly slow rather than fast ones occurred on initial trials. In Experiment 2, we contrasted the frequency effect and the primacy effect: In runs with frequent slow velocities, mainly fast ones occurred earlier, whereas in runs with infrequent slow velocities, mainly slow ones did so. Lack of differences of ratings in the two conditions suggests that the two effects canceled each other. In Experiment 3, when mainly frequent velocities occurred earlier, the conventional frequency effect was obtained. We conclude that the conventional frequency effect represents a combination of the primacy effect and the pure frequency effect.
\end{abstract}

When judging some property of an object or a person, people typically use a reasonably restricted set of three to five categories. Such absolute judgments have been found to depend substantially on the frequency of occurrence of the stimuli to be judged (e.g., Marks, 1968; Parducci, 1956; Poulton, 1968; Stevens, 1971). In particular, identical stimuli receive higher ratings in a positively skewed distribution (with a greater proportion of small stimuli) than in a negatively skewed distribution in which large stimuli are more frequent (see, e.g., Parducci, 1963; Parducci \& Perrett, 1971). This frequency effect increases

Parts of this study were presented at the 12th and 14th Annual Meetings of the International Society for Psychophysics and at the 19th European Conference on Visual Perception. The experiments were conducted at Institut für Arbeitsphysiologie, Dortmund, Germany, while Alexander Sokolov was an Alexander von Humboldt Fellow; during the preparation of the manuscript he was supported by the German Research Council (DFG). Marina Pavlova and Alexander Sokolov are on leave from the Institute of Psychology, Russian Academy of Sciences, Moscow. We thank Niels Birbaumer, C. Richard Cavonius, Dieter Heyer, and Rainer Mausfeld for support, Ehtibar Dzhafarov, Gert Haubensak, and Viktor Sarris for stimulating discussions, and Peter Dillmann for technical assistance. We appreciate Daniel Algom, an anonymous reviewer, and especially John C. Baird for valuable comments and advice on an earlier version of the manuscript. Correspondence concerning this article should be addressed to A. Sokolov, Institute of Medical Psychology and Behavioral Neurobiology, MEG-Center, University of Tübingen, Gartenstr. 29, D-72074 Tübingen, Germany (e-mail: alexander. sokolov@uni-tuebingen.de). dramatically as the number of different stimuli increases or the number of available categories decreases (Parducci \& Wedell, 1986; Wedell, 1984).

There are two well-elaborated accounts of the frequency effect. The range-frequency theory (e.g., Parducci, 1963; Parducci \& Wedell, 1986) assumes that the absolute judgment represents a compromise between a subject's tendencies to judge each stimulus relative to the endpoints of the stimulus range (the range principle) and to assign the same number of stimuli to each category (the frequency principle). When the frequency of occurrence of the small (or large) stimuli is greater, subjects are expected to redistribute stimuli into the higher (or lower) categories (Parducci, 1983; Stevens \& Galanter, 1957).

More recently, Haubensak $(1982,1985,1992)$ proposed the consistency model, which provides an alternative account of the frequency effect. It is supposed that if there are no right and wrong answers, subjects tend to be "internally consistent" (Haubensak, 1992, p. 304), assigning the same stimulus to the same category. When stimuli are presented randomly without replacement, more frequent stimuli are more likely to occur on the initial trials (Haubensak, 1992, Figure 1; Johnson \& Kotz, 1977). A subject's scale becomes rapidly centered on the range of the stimuli that occur early in a presentation series. This results in the assignment of the rest of stimuli to the higher or lower categories (the primacy effect). Therefore, the presentation order of stimuli (especially with re- 
spect to those presented on the early trials of a run), rather than their frequency of occurrence per se, might generate a frequency-like effect on absolute judgments.

Two bodies of evidence on scale development and on transfer effects favor the consistency over the rangefrequency account of the frequency effect.

(1) The scale of a subject was found to assume its essential form, caused by contextual frequency, fairly quickly (for 8-16 initial trials; Haubensak, 1985; Parducci \& Wedell, 1986). Related evidence was reported by Schüßler (1981): When only the smallest and the largest stimuli were presented as background, the frequency effect was greatly diminished, because these endpoint stimuli allowed subjects to center their scale on the whole stimulus range at the very beginning of a run.

Because the frequency effect emerges at such an early stage of the experiment, an explanation based on the subjects' tendency to use the categories equally often does not seem applicable to these results. At the start of an experiment, subjects are not aware of the actual overall frequencies of the stimuli (Haubensak, 1992) or of the frequencies with which the categories will be used.

Furthermore, after the experiment, subjects typically have difficulty indicating the correct number of different stimuli presented. Parducci (1963) observed very large variability of such reports, with only $10 \%$ correct. Obviously, the subjects are unlikely to have access to the stimulus frequencies without knowing how many distinct stimuli have been shown. According to the range-frequency theory, they may also have an intuition about how often each category has been used. It was found, however, that providing the subjects with a cumulative record of their own response frequencies yielded only a slight increase of the frequency effect (Parducci, 1963; Parducci \& Perrett, 1971). If subjects tended to employ the categories equally often, such a feedback would produce a more pronounced effect, because it would improve monitoring of the frequency of a category used.

(2) Research on transfer effects (Poulton, 1979) suggests that the judgment scale is resistant to shifts in the stimulus frequency and stimulus range (Di Lollo, 1964; Guilford, 1954; Parducci, 1954; Ward \& Lockhead, 1970). Haubensak (1989) reported that after subjects judged either lower or upper subranges of different-sized stimuli, they were able to only partially adjust their scales to the whole stimulus range. Even when subjects were directly instructed to modify their scales, the scales tended to retain an initial form.

With frequency shifts, the scale adjustment proceeds more readily even without informing the subjects about the shift but still takes a long time to be completed. After subjects judged positively (or negatively) skewed sets, their ratings with postshift opposite frequency skews coincided, starting with the second run and lasting as long as three additional runs (Wedell, 1984, chap. 4). The convergence of postshift ratings indicates that a scale is only partly adjusted to the new contextual frequencies. More- over, in all, $25 \%$ of the subjects showed a complete scale adjustment (Parducci, 1992; Wedell, 1984). When the initial trials are unrepresentative of the overall distribution, a reliable adjustment of the scale to the actual skewing occurs only beyond 40 trials (Parducci, 1992).

Thus, evidence both on the transfer effects and on the time course of scale development favors the consistency model account of the frequency effect over that suggested within the range-frequency framework. Some modifications of the latter model seem capable of explaining the early scale development. For example, the retrieval consistency model (Parducci \& Wedell, 1986) assumes a tendency for consistent identification - that is, subjects "repeat the judgment previously applied to the stimulus" (Parducci, 1959, p. 246). The stimulus frequencies are supposed to be represented in implicit memory, although their retrieval is restricted to the 12 most recent trials (Parducci, 1992; Parducci, Knobel, \& Thomas, 1976). However, with such a restricted retrieval set, the model faces a difficulty when explaining the long-term effect of initial presentations in transfer studies. The incomplete scale adjustment following contextual shifts suggests that the stimuli of the preshift run are still effective for the postshift trials. In contrast, the censistency framework, which requires no counting of the stimulus frequencies at all (Haubensak, 1992), seems to more comprehensively deal with these findings.

With a standard randomization of trials in the laboratory setting, both the presentation order of stimuli and their frequency of occurrence might affect judgments simultaneously and in similar ways, because more frequent stimuli occur earlier. Therefore, theoretically there are three possibilities for explaining the conventional frequency effect (Sokolov \& Ehrenstein, 1996): (1) frequency of occurrence (range-frequency theory), (2) presentation order (consistency model), or (3) a combined effect of both factors. The present study was intended to experimentally disentangle these possibilities.

Typically, absolute judgments of size have been used to examine the frequency effect (e.g., Haubensak, 1992; Parducci \& Wedell, 1986), although similar contextual effects have been documented in many sensory modalities and stimulus dimensions, ranging from lifted weights to sweetness of solutions (e.g., Algom \& Marks, 1990; Di Lollo, 1964; Schifferstein \& Frijters, 1992). In this study, we contrast the predictions of range-frequency and consistency accounts by using judgments of visual velocity. The methodological approach consisted of manipulating the statistical structure of stimulus sets - that is, their skewing and the probability for different stimulus velocities to be presented on the initial trials of a run.

In a first experiment, we tried to obtain a frequencylike primacy effect while keeping the actual frequencies of occurrence for stimulus velocities in the sets equal. Their presentation order, however, was biased in such a way that it mimicked the presentation order of either positively or negatively skewed source distributions. That is, 
in the former case, on the initial trials of a run, slow velocities were mainly presented, whereas in the latter, fast velocities were mainly presented.

A second experiment was designed to determine whether with actual different-skewed stimulus sets, there are two distinct (primacy and frequency) effects on absolute judgments. For this purpose, we contrasted the two factors. That is, the order of a positively skewed set was adjusted so that it resembled the order of a negatively skewed source distribution (with mainly fast velocities being presented on initial trials), and vice versa.

Finally, we conducted a third experiment with the same different-skewed sets of stimulus velocities without biasing their presentation order. There were two reasons for this experiment. First, we sought to provide a control for the previous experiments examining whether the conventional frequency effect occurs with judgments of visual velocities. Second, and most important, we tried to isolate the primacy and frequency effects, if any, with different-skewed sets.

\section{EXPERIMENT 1 \\ Presentation Order Versus Equal Frequency of Occurrence}

According to the consistency model, the presentation order of equal-frequent stimuli, which only resembles the order of either positively or negatively skewed distributions, should generate a frequency-like primacy effect. According to the range-frequency expectations, the ratings should be indifferent as to the variable presentation order, because the actual frequencies of the stimuli remain the same.

\section{Method}

Subjects. Sixteen male and 16 female observers (mean age, 25.8 years) with normal or corrected-to-normal vision participated. They were paid and were naive as to the aim of the research. The subjects were run individually.

Stimuli. Five stimulus velocities $(3 \% \mathrm{sec}, 4.5 \% \mathrm{sec}, 6 \% \mathrm{sec}$, $7.5 \% \mathrm{sec}$, or $9 \% \mathrm{sec})$ were used. Horizontal motion of a single light $\operatorname{dot}\left(0.15^{\circ}\right.$ in diameter, with a luminance of $\left.60 \mathrm{~cd} / \mathrm{m}^{2}\right)$ was presented on a fast-phosphor 14-in. monitor within a rectangular aperture $\left(23^{\circ} \times 3^{\circ}\right)$ created by cutting an opening in the black cardboard that covered the screen.

Table 1

Stimulus Velocities (\% $/ \mathrm{sec})$ as a Combination of Duration and Extent of Motion

\begin{tabular}{cccc}
\hline & \multicolumn{3}{c}{ Extent $\left(^{\circ}\right)$} \\
\cline { 2 - 4 } Duration $(\mathrm{sec})$ & 2 & 2.5 & 3 \\
\hline 0.267 & 7.5 & - & - \\
0.278 & - & 9 & - \\
0.333 & 6 & 7.5 & 9 \\
0.500 & - & - & 6 \\
0.556 & - & 4.5 & - \\
0.667 & 3 & - & 4.5 \\
0.833 & - & 3 & - \\
\hline
\end{tabular}

Note-In a single experiment, each combination resulting in a particular stimulus velocity was used equally often.
The dot appeared and started to move $0.5^{\circ}$ below fixation at an observation distance of $57 \mathrm{~cm}$. In order to prevent adaptation to motion direction and a reliance, while velocity was being judged, on spatial or temporal cues (Mandriota, Mintz, \& Notterman, 1962), the velocities were presented in a balanced combination of motion duration, extent, and direction (left/right; no more than two successive trials in the same direction). Table 1 shows the combinations of stimulus duration and extent of motion that resulted in the five stimulus velocities used in the study.

Generation of experimental sequences. The following algorithm was used to generate two types of experimental sequences with equal-frequent stimuli that mimicked the presentation order of either positively or negatively skewed source distributions. First, the relative frequencies of the five stimulus velocities in the source distributions were adjusted so as to be identical to those used in Parducci and Wedell (1986, Table 2, p. 499: 20-14-8-4-4 for positive and 4-4-8-14-20 for negative skewing, respectively). To get a sufficient number of trials, all the frequencies were increased by a factor of 2.5 (see Table 2 below; multiplication only slightly decreased the original skewing value- $-0.940 \mathrm{vs.} 0.922$ in the source distributions).

Second, each of these two source distributions was randomized with a standard computer-based procedure. For the sake of intraskewing comparison, two different random sequences of trials were created from both positively and negatively skewed source samples. Finally, to get equal frequencies of occurrence for the stimuli in each sequence, we selected for presentation only the first 10 repetitions of each stimulus velocity, counting from the beginning of the series (Table 2).

Thus, every presentation sequence consisted of 50 trials that were ordered as to the respective source distribution. In the sequences derived from the positively (or negatively) skewed source distribution, mainly slow (or fast) velocities were exposed on initial trials. The frequency skewing in both presentation sequences was equal to zero.

Procedure. Two experimental sequences were presented to different groups of 16 subjects each ( 8 males and 8 females). Within the groups, two different positively biased or two negatively biased presentation orders were used for different subjects.

An experimental trial began with an auditory warning tone, followed after a 500 -msec interval by a target that moved at one of five stimulus velocities. The subjects were asked to fixate a point in the middle of the opening and to pursue the target while it moved. A head-and-chin rest restricted head movements. The subjects had to judge each stimulus velocity, using three verbal categories (slow, medium, and fast). They were informed about the stochastic combination of duration and distances, which produced different velocities. The subjects were asked to rely strictly on their impression of velocity. They were informed that there were no formal criteria for right and wrong answers. The subjects performed the task without preliminary acquisition trials.

\section{Results}

Figure 1 (solid lines) shows the ratings of five stimulus velocities averaged across the subjects of each experimental group. The data indicate that the mean ratings of identical (and equal-frequent) velocities are systematically higher for positively than for negatively biased sequences. A two-way analysis of variance (ANOVA) performed on individual ratings of stimulus velocities in the two groups revealed a highly significant main effect of presentation order $[F(1,15)=10.562, p<.005]$.

Thus, depending on the presentation order, equal frequencies of stimulus occurrence produce an effect similar to that typically obtained with real different-skewed sets of stimuli (e.g., Parducci \& Wedell, 1986). This supports the prediction of the consistency model. However, 
Table 2

Stimulus Frequencies in the Source and Presentation Sets

\begin{tabular}{|c|c|c|c|c|c|}
\hline \multirow[b]{2}{*}{ Set Skewing } & \multicolumn{5}{|c|}{ Velocity $(\% / \mathrm{sec})$} \\
\hline & 3 & 4.5 & 6 & 7.5 & 9 \\
\hline \multicolumn{6}{|c|}{ Experiment 1} \\
\hline \multicolumn{6}{|l|}{ Source distributions } \\
\hline Positive & 50 & 35 & 20 & 10 & 10 \\
\hline Negative & 10 & 10 & 20 & 35 & 50 \\
\hline \multicolumn{6}{|l|}{ Actual occurrence } \\
\hline None + "positive" order* & 10 & 10 & 10 & 10 & 10 \\
\hline None + "negative" order $\dagger$ & 10 & 10 & 10 & 10 & 10 \\
\hline \multicolumn{6}{|c|}{ Experiment 2} \\
\hline \multicolumn{6}{|l|}{ Source distributions } \\
\hline Positive & 100 & 70 & 40 & 20 & 20 \\
\hline Negative & 20 & 20 & 40 & 70 & 100 \\
\hline \multicolumn{6}{|l|}{ Actual occurrence } \\
\hline Negative + "positive" order* & 4 & 4 & 8 & 14 & 20 \\
\hline Positive + "negative" order $\dagger$ & 20 & 14 & 8 & 4 & 4 \\
\hline \multicolumn{6}{|c|}{ Experiment 3} \\
\hline \multicolumn{6}{|l|}{ Actual occurrence } \\
\hline Positive & 20 & 14 & 8 & 4 & 4 \\
\hline Negative & 4 & 4 & 8 & 14 & 20 \\
\hline
\end{tabular}

*"Positive" order, mainly slow velocities occur earlier. "'Negative" order, mainly fast velocities occur earlier.

the finding poses a problem for the range-frequency theory, because the effect occurs without differences in the frequency of stimulus occurrence in the two sets.

Computer simulations of the consistency model (Haubensak, 1992; available as software from the author) revealed good agreement between predicted and observed results (Figure 1, dashed lines). The model has two parameters: $b$, long-term memory (or learning), and $f$, forgetting. The choice of their particular values $(b=0.01$ and $f=0.1$ ) used in all simulations throughout the study was determined by empirical estimations, using data reported by Parducci and Wedell (1986) (Haubensak, 1992, p. 305). No significant differences between the judgments of real and "artificial" subjects were found $[F(1,15)=0.217$ and 0.604 for positively and negatively biased sequences, respectively, $p s>.05]$.

Moreover, our data suggest a possibility for an even stronger primacy effect under certain conditions. Consider the group of subjects who judged positively biased sets. Despite the common positive bias, the presentation order of sets varied between the two subgroups of this group. In one set, faster velocities $(6 \% \mathrm{sec}$ and $9 \% \mathrm{sec})$ occurred only beyond the sixth trial, whereas in the other, those velocities were by chance presented in four of the five initial trials. The ratings were found to be lower in the latter than in the former subgroup $[F(1,7)=6.645$, $p<.05$; see Figure 2], most likely because the latter sequence more closely resembled a negatively rather than a positively biased sequence. Hence, the mean ratings of the whole positive group shown in Figure 1 could be even higher if, in one of its subgroups, the rapid velocities did not occur early in the run. In agreement with the consistency model, this dependence of ratings on the stimuli presented within 5-6 initial trials suggests early formation of the subject's scale and a remarkable sensitivity of judgments to the presentation order of equal-frequent stimuli.

\section{Discussion}

Experiment 1 shows that absolute judgments of identical velocities are of a greater magnitude when the presentation order of sets with equal-frequent velocities is derived from positively skewed source distributions rather than from negatively skewed ones. Therefore, the presentation order of stimuli produces a primacy effect that is similar to the conventional frequency effect. This extends the previous evidence from Haubensak (1989) on the primacy effect: With scale transfer, he showed that the primacy effect continued across a postshift series after the range of stimuli was changed. We obtained the primacy effect within a single run.

The results seem to favor the consistency model account of the conventional frequency effect (Haubensak, 1992) over that provided within the range-frequency framework (Parducci \& Wedell, 1986). However, such a conclusion has to be made with caution, because we examined only sets with equal frequencies of stimulus occurrence. Actually, in the case of real differently skewed distributions, when both factors are present, the (pure) frequency effect may still be confounded with the primacy effect.

There is yet another reason to try real different-skewed stimulus sets. Sander and Sarris (1997) have recently criticized the range-frequency theory because of its failure to predict a scale generalization in a two-stimulustwo-choice paradigm. They employed, however, bimodal stimulus sets, whereas the theory seems to apply primarily to unimodal distributions of stimuli (see Parducci $\&$ Wedell, 1986). Since the sets we used in Experiment 1 were also not unimodal (they had neither peak stimulus frequency nor even differences in the stimulus frequencies

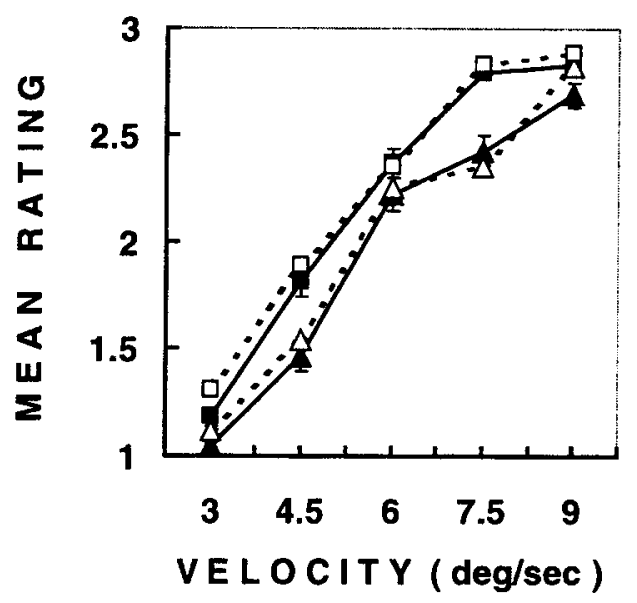

Figure 1. Mean ratings of velocity for positively (squares) and negatively (triangles) biased sets of equal-frequent stimuli ( $E x-$ periment 1). Each experimental point is based on data from 16 subjects; vertical bars, $\pm S E$. Closed and open symbols show experimental data and computer simulations of the consistency model, respectively. 


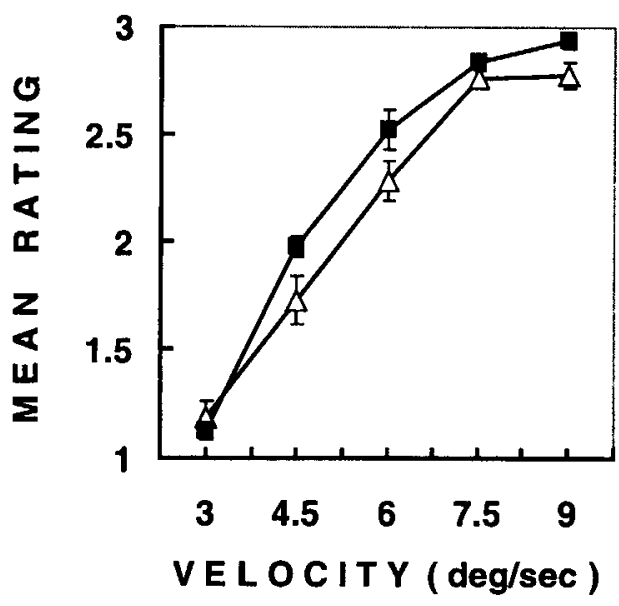

Figure 2. Mean ratings of velocity for two positively biased sets (Experiment 1). Each experimental point is based on data from 8 subjects. Data are shown for sets in which faster velocities $(6 \% \mathrm{sec}$ and $9 \% / \mathrm{sec}$ ) occurred relatively earły (open triangles) or late (closed squares).

within and between sets), a comparison of the results with the range-frequency expectations might be considered unjustified.

Therefore, although in Experiment 1 we established the frequency-like primacy effect, it remains to be determined whether with different-skewed stimulus sets, there is only a primacy effect or a combination of two effects.

\section{EXPERIMENT 2 Presentation Order Versus Frequency of Occurrence}

In this experiment, we biased the orders of differentskewed sets in the direction opposite to that defined by their skewing. That is, in the set with positive skewing, mainly fast (instead of slow, as with a standard randomization) velocities occurred on the initial trials of a run, although their proportion in a total sequence was lower. Conversely, in the negatively skewed set, mainly infrequent slow velocities were presented earlier. This methodological approach enabled us to contrast the presentation order of stimuli and their frequency of occurrence within a sequence, whereas typically, both factors are likely to be confounded.

If the frequency of stimulus occurrence in differentskewed sets is the only factor that determines the conventional frequency effect (which would thus be the pure frequency effect), then despite the presentation orders, identical velocities should be rated as more fast in the positively skewed sets than in the negatively skewed ones. Alternatively, if the presentation order of stimuli is critical, the ratings should be higher in the positively biased sequences than in the negatively biased sequences, regardless of actual skewing of stimulus frequencies. If both factors operate in opposite directions, the outcome should depend on the relative contribution of each factor.

\section{Method}

Subjects. Thirty-two paid observers (16 male and 16 female; mean age, 28.5 years) participated. Each had normal or correctedto-normal vision, and none had taken part in Experiment 1

Stimuli and Procedure. The experimental setup, stimuli, and procedure were essentially the same as those in Experiment 1. The main difference was that, instead of an equal number of stimulus velocities, the sets included different proportions of distinct velocities. For the sake of comparison, these stimulus frequencies were chosen so as to be identical to those used by Parducci and Wedell (1986). However, unlike Parducci and Wedell, we biased the presentation order of each set in the direction opposite to that caused by its actual skewing. In our experiment, rare stimuli had a greater probability to occur on the initial trials of a run. Every presentation sequence consisted of 50 trials (see Table 2).

Generation of experimental sequences. Presentation orders were produced with the following algorithm. First, the relative frequencies of stimulus velocities in our source distributions were adjusted so as to equal those used by Parducci and Wedell (1986, Table 2, p. 499). To provide a sufficient number of trials, all the frequencies were multiplied by five (see Table 2). The skew values of our source distributions were then equal to \pm 0.917 . Second, each of these source distributions was twice randomized by a standard computer-based procedure. Two different positively and two negatively skewed source sequences were produced.

Finally, unlike Experiment 1, from the negatively skewed source sequences, we selected for presentation each stimulus velocity with the frequency (counting from the start of a sequence) that exactly corresponded to the positively skewed set of Parducci and Wedell (1986; see Table 2). Note that the order of their presentation was still determined by a particular source-set skewing, whereas actual stimulus frequencies were reversed, as compared with the source skewing. The actual frequencies of occurrence for stimulus velocities selected from the positively skewed source sequences were equal to those in the negatively skewed set of Parducci and Wedell (few slow and many fast velocities). Yet, their presentation order still corresponded to that produced by the positive skewing of the source set.

Thus, such a manipulation resulted in presentation orders of sequences that were opposite to those contingent on their particular skew. Specifically, in the sequences with a greater proportion of

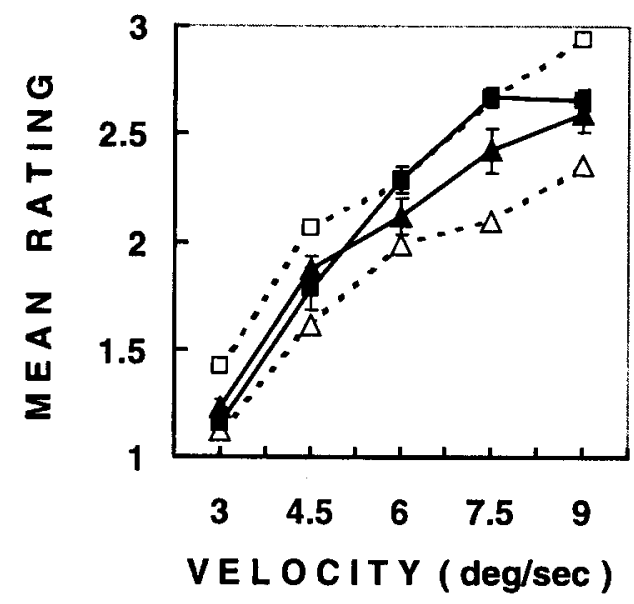

Figure 3. Mean ratings of velocity for negatively skewed/positively biased (squares) and positively skewed/negatively biased (triangles) sets (Experiment 2). Each experimental point is based on data from 16 subjects. Closed and open symbols show experimental data and computer simulations of the consistency model, respectively. 
slow (positive skewing) or fast (negative skewing) velocities, mainly fast or slow ones occurred on the initial trials.

\section{Results}

Figure 3 (solid lines) shows the ratings of stimulus velocities averaged across the subjects of each experimental group. The ratings of the two groups are virtually the same. There is an indication neither of the frequency effect nor of the primacy effect. A two-way ANOVA did not reveal any significant difference between the two groups $[F(1,15)=1.334, p>.05]$. The interaction of group and velocity approached significance $[F(4,15)=2.305, p<$ $.07]$, suggesting a slight preference for the primacy effect beyond moderate velocity $(6 \% \mathrm{sec})$. Two stimulus velocities $\left(6 \% \mathrm{sec}\right.$ and $\left.7.5^{\circ} / \mathrm{sec}\right)$ were rated faster when presented in the positively biased sets than when presented in the negatively biased sets $[t(30)=2.256, p<.06$, and $t(30)=2.815, p<.02$, respectively]. There were no differences between the subgroups that rated different positively and negatively biased sets $[F(1,7)=1.165$ and $0.727, p$ s $>.05]$.

Computer simulations of the consistency model with the sets used in the present experiment yielded a marked discrepancy between the predicted and the experimental results (Figure 3, dashed lines). The model predicted a rather strong primacy effect across the entire velocity range, whereas only a slight preference for this effect was obtained for faster velocities. The results of an ANOVA indicated significant differences between the predicted and the obtained ratings within the two groups $[F(1,15)=$ $9.916, p<.01$, and $F(1,15)=4.508, p<.05$, for positively and negatively biased sets, respectively].

Similarly, the results do not conform to the expectations based on the range-frequency theory. With the sets of variable skewing, we failed to obtain the conventional frequency effect (higher ratings for the positively skewed set). This is noteworthy, because we used stimulus sets with exactly the same skewing and number of stimuli and categories as those employed by Parducci and Wedell (1986, Experiment 1).

\section{Discussion}

The purpose of this experiment was to disentangle the primacy and the frequency effects and to clarify their impact on absolute judgments. Our findings strongly support the assumption that the presentation order of stimuli and their frequency jointly produce the conventional frequency effect.

The main difference of our experiment from that of Parducci and Wedell (1986) was that the order of their presentation sequences was contingent on the set skewing, whereas in the present experiment, it was reversed. The order of positively skewed sets was derived from the negatively skewed source distribution, and vice versa. Despite the different frequency skews used, a strong opposite effect of the presentation order resulted in the same ratings of the groups (Figure 3). A slight preference for the primacy effect with faster velocities suggests that this effect is at least as powerful as the pure frequency effect.
Further research is needed to determine whether the two effects are sensory dependent, dependent on decisionmaking, or response dependent (see Wedell, 1990).

The present findings seem to be at odds with those reported by Parducci (1992). He had subjects rate stimulus sets in which the initial trials were unrepresentative of the overall stimulus distribution. Similarly, in our experiment, the rare stimuli had a greater probability to occur early in the series. Thus, both manipulations may be viewed as a frequency shift within a run. Parducci (1992) found a reliable adjustment of the subject's scale to the actual overall skewing, despite the early occurrence of rare stimuli. In his study, however, only three initial stimulus presentations were modified; still, a considerable number of trials (more than 40 ) were needed for the adjustment to occur. Our cumulative data were collected using a more extensive modification of the initial portion of a run. That the ratings of the two groups were essentially the same (Figure 3 ) also suggests a remarkable, although still incomplete, adjustment of their scales. Thus, the evidence for the scale adjustment and its considerable time course found in both studies support our assumption that the frequency of stimuli and their presentation order jointly affect absolute judgments in the conventional frequency effect.

In Experiment 2 we contrasted these two effects with different-skewed stimulus sets but did not isolate them operationally. In addition, there is a lack of evidence for the conventional frequency effect with visual velocity. The next experiment was conducted to resolve these issues.

\section{EXPERIMENT 3 Contingent Presentation Order and Frequency of Occurrence}

If the conventional frequency effect occurs with judgments of visual velocities, the ratings of identical velocities should be higher when the stimuli are presented in a positively skewed set than when they are presented in a negatively skewed set. It is important that the presentation order of stimulus velocities in the two sets is contingent on the respective set skewing.

\section{Method}

Subjects. Two groups of 16 paid observers (equal number of males and females; mean age, 29.9 years) took part in the experiment. They had normal or corrected-to-normal vision, and none had participated in the previous experiments.

Stimuli and Procedure. The experimental setup, stimuli, and procedure were the same as those in Experiments 1 and 2. The only difference from Experiment 1 was that now we used different-skewed stimulus sets identical to those in Experiment 2. However, in contrast to Experiment 2, we did not introduce any bias when randomizing the sets.

Generation of experimental sequences. Two positively and negatively skewed stimulus sets were used. The frequencies of occurrence for stimulus velocities in these sets were identical to those in Parducci and Wedell (1986). Both sets were randomized (without bias), using a standard computer-based procedure. Unlike Experiment 2, but as in the Parducci and Wedell study, the presentation orders of the velocities were statistically determined by their fre- 
quencies of occurrence in the two sets (Haubensak, 1992; Johnson \& Kotz, 1977).

Two different positively and two negatively skewed sequences (of 50 trials each) were created. The positively skewed sequences consisted of a greater proportion of slow velocities, with mainly these velocities being presented on initial trials. In the negatively skewed sequences, the proportion of fast velocities was greater, and mainly these velocities occurred early in the run (Table 2).

\section{Results}

Figure 4 (solid lines) represents the mean ratings of stimulus velocities averaged across the subjects within each experimental group. Identical stimulus velocities were rated as much faster when presented in the positively than when presented in the negatively skewed sets. The results of a two-way ANOVA yielded a highly significant main effect of the group $[F(1,15)=62.117, p<$ $.001]$. No differences in the ratings between subgroups within each group were found.

Computer simulations of the consistency model also show good correspondence between predicted and obtained results (see Figure 4, dashed lines). An ANOVA did not reveal any difference in the simulated and the experimental ratings within the groups $[F(1,15)=1.369$ and 1.039 , for positively and negatively skewed sets, respectively, $p \mathrm{~s}>.05]$.

\section{Discussion}

Experiment 3 clearly demonstrates that as with a wide variety of other stimulus properties, the conventional frequency effect occurs with absolute judgments of visual velocities. Therefore, the present findings cannot be attributed to the specific stimuli employed.

Although the only difference between Experiment 3 and the previous one consisted in the presentation order, this difference gave rise to strikingly divergent results. In Ex-

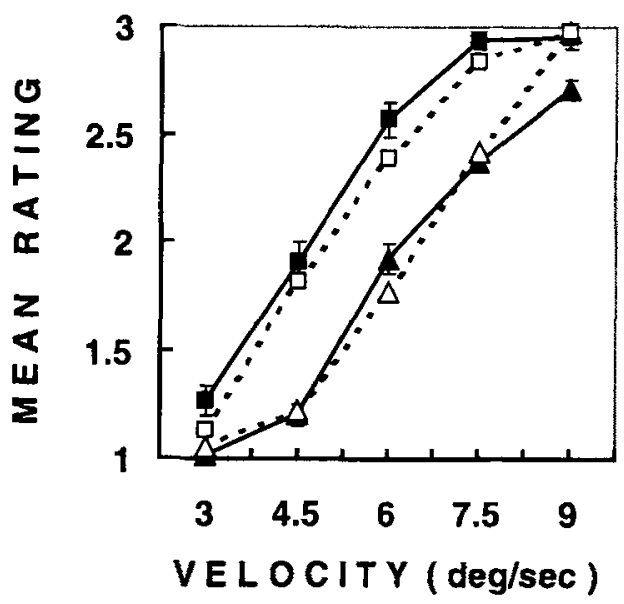

Figure 4. Mean ratings of velocity for positively (squares) and negatively (triangles) skewed sets with orders contingent on their skew (the conventional frequency effect, Experiment 3). Each experimental point is based on data from 16 subjects. Closed and open symbols show experimental data and computer simulations of the consistency model, respectively.

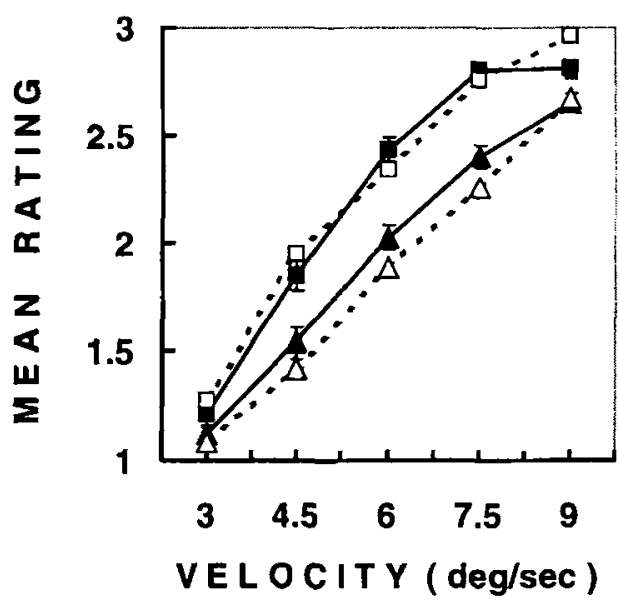

Figure 5. Mean ratings of velocity for positively (squares) and for negatively (triangles) biased sets (the primacy effect, Experiments 2 and 3). Each experimental point is based on data from 32 subjects. Closed and open symbols show experimental data and computer simulations of the consistency model, respectively.

periment 2 , we obtained essentially the same ratings for different-skewed sets, whereas in Experiment 3 these ratings were distinct. Because in both experiments, we used a between-subjects design, they can be viewed as a unified experimental arrangement. This allows one to isolate the primacy and pure frequency effects. To elucidate the primacy effect (Figure 5, solid lines), we pooled the ratings of the groups with the positively biased presentation order (negatively and positively skewed sets in Experiments 2 and 3 , respectively) versus those with the negatively biased order. To segregate the pure frequency effect (Figure 6, solid lines), we averaged the group ratings for positively skewed versus negatively skewed sets in these experiments.

Figure 5 shows that identical velocities were rated as more fast (or slow) when mainly slow (or fast) velocities occurred on the initial trials. Figure 6 indicates that the way they were rated depended on whether slow or fast velocities had a greater frequency of occurrence. Mean magnitude of the two effects was .27 of the category size in the former and .21 in the latter cases. A two-way ANOVA revealed main effects for both presentation order and stimulus frequency $[F(1,31)=64.160$ and 19.994 , respectively, $p s<.001]$. The primacy effect was stronger with faster stimulus velocities, whereas the pure frequency effect was stronger with slower ones: The presentation order $X$ velocity and the frequency $X$ velocity interactions were significant $[F(4,31)=5.105$ and 4.029 , respectively, $p s<.01]$. The simulations with the consistency model produce a fairly good fit to the primacy effect $[F(1,31)=$ 0.422 and 2.234 , for positively and negatively biased orders, $p s>.05$; Figure 5, dashed lines]. Yet, there is a lack of such correspondence with the pure frequency effect $[F(1,31)=5.718$ and 9.024 for positive and negative skewing, respectively, $p \mathrm{~s}<.02$; Figure 6 , dashed lines]. Thus, the comparison of the findings of Experiments 2 


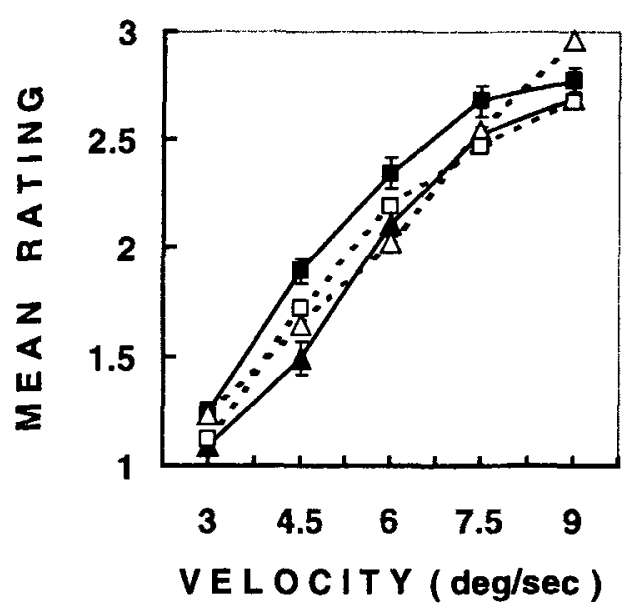

Figure 6. Mean ratings of velocity for positively (squares) and for negatively (triangles) skewed sets (the pure frequency effect, Experiments 2 and 3). Each experimental point is based on data from 32 subjects. Closed and open symbols show experimental data and computer simulations of the consistency model, respectively.

and 3 strongly supports our assumption that the primacy and the pure frequency effects usually operate simultaneously and in the same direction.

\section{GENERAL DISCUSSION}

In a set of three experiments, we have shown that in accord with predictions of Haubensak's consistency model, the initial portion of a series does affect absolute judgments. Therefore, the wording of the conventional frequency effect reported by Parducci and his colleagues (e.g., Parducci, Calfee, Marshall, \& Davidson, 1960; Parducci \& Perrett, 1971) needs to be reformulated: Identical stimuli receive higher ratings when presented among many small stimuli than when presented among many large stimuli if and only if these frequent stimuli mainly occur on the initial trials.

We have isolated the primacy and the pure frequency effects that usually combine to produce the conventional frequency effect. It is known that the conventional effect becomes stronger with an increasing number of distinct stimuli and a decreasing number of categories (Parducci $\&$ Wedell, 1986; Wedell, 1984). Given the primacy and frequency origins of the conventional effect, these variables might affect one or both components simultaneously.

Both the primacy and the pure frequency effects are long term: An inspection of Figures 1, 5, and 6 indicates that over the entire stimulus range, each experimental curve is nearly uniformly displaced relative to the other. Therefore, they differ from the sequential, or recency, effects in absolute judgments (see, e.g., Jesteadt, Luce, \& Green, 1977; Schifferstein \& Frijters, 1992), which are considered short term, lasting a maximum of eight trials back from the currently presented stimulus (Lockhead, 1984). Evidence on the long-term primacy effect with equal-frequent stimuli might be of use for research based on rating methods. For example, in obtaining ratings for multidimensional scaling, the initial trials are likely to affect the judgment of a current stimulus and, hence, to produce distortions of the resulting subjective space. To avoid this, one might want to control for stimulus frequencies by appropriately reducing, in the remainder of a run, the occurrences of stimuli that have been presented earlier.

With the primacy effect, the early trials more strongly affect the judgments of rarely presented stimuli $[F(1,15)=$ 26.944 and 37.744 for main effects of order, and $F(4,15)=$ 7.4 and 9.504 for order $\times$ velocity interactions, $p s<.001$; Figure 7]. The pure frequency effect is more pronounced for those stimuli that occur mainly on the last trials $[F(1,15)=9.584$ and $9.838, p s<.01$, for main effects of skewing, and $F(4,15)=1.652, p>.05$, and $16.757, p<$ .01 , for skewing $\times$ velocity interactions; Figure 8 ]. Thus, the combination of the two effects leads to the conventional frequency effect (Figure 4), whereas their op-

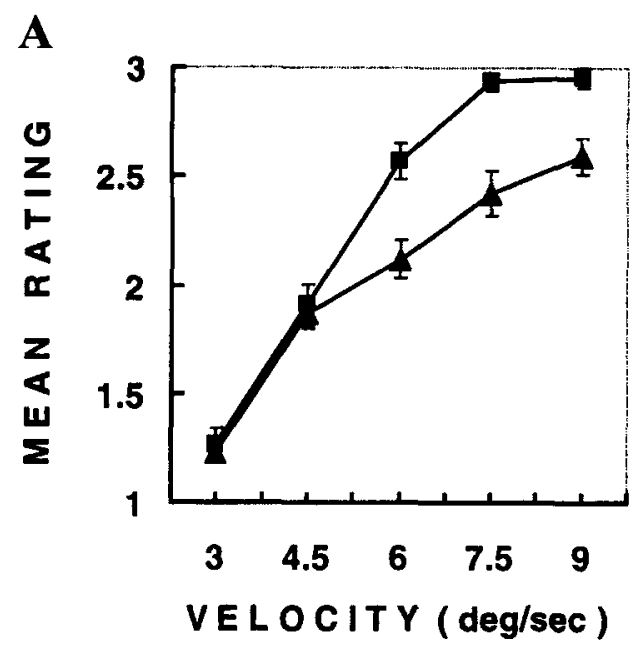

B

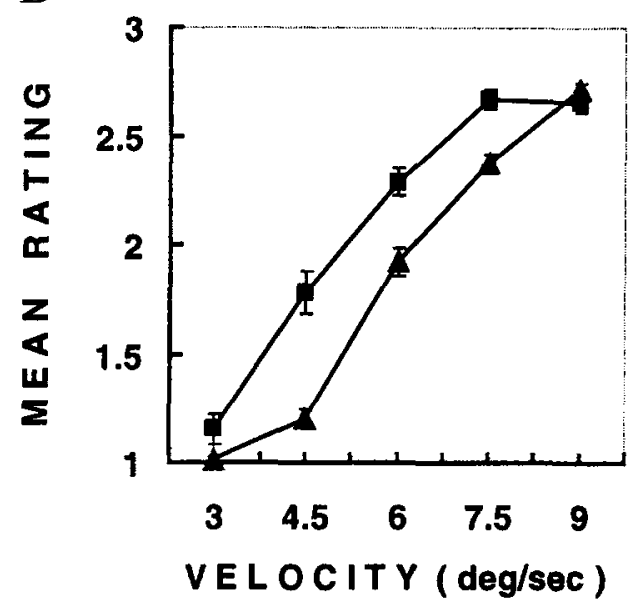

Figure 7. Mean ratings of velocity with (A) positively and (B) negatively skewed sets (the primacy effects, Experiments 2 and 3). Data for positively (squares) and for negatively (triangles) biased presentation orders. Each experimental point is based on data from 16 subjects. 

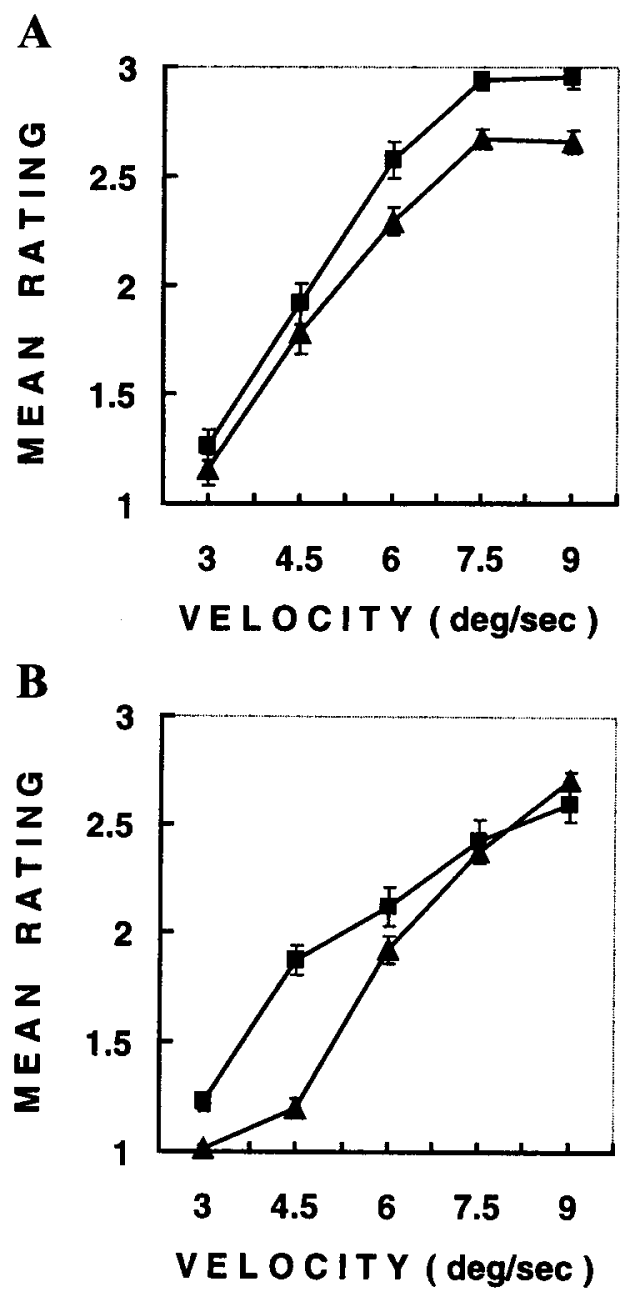

Figure 8. Mean ratings of velocity with (A) positively and (B) negatively biased sets (the pure frequency effects, Experiments 2 and 3). Data for positive (squares) and for negative (triangles) frequency skews. Each experimental point is based on data from 16 subjects.

posite combination results in essentially identical ratings (Figure 3).

Our findings suggest that the judgment scale is adaptable to the changing context for judgment. The results of Experiment 1 confirm the claim of the consistency model that the subject's scale develops fairly rapidly (Haubensak, 1992). The lack of differences in ratings found in Experiment 2, however, contradicts the assumption that once established, the scale tends to retain its form over the remainder of the run. The strong effect of initial trials on absolute judgments indicates that in estimating stimuli, people follow the law of small numbers (Parducci \& Wedell, 1986; Tversky \& Kahneman, 1974). In accordance with this law, subjects assume that a small random sample is more representative of the whole set than a sample derived on the basis of the conmon statistical rules of sampling. On the other hand, the pure frequency effect obtained in Experiments 2 and 3 suggests that, in the long run, people tend to be "realists," adjusting their scales to the changing context. Apparently, this holds only for the sets that contain clearly distinct stimuli. These tendencies may change with sets of barely distinguishable stimuli. In this case, people are likely to respond as if they relied on the assumption that stimuli are normally distributed (Erlebacher \& Sekuler, 1971).

Although this study was not specifically concerned with velocity perception as such, our data suggest some implications for daily life situations. For example, when exiting the highway, one decreases his or her speed. Yet, the speed of other cars still appears extremely slow. This might occur because the preceding velocities were skewed toward the high-velocity range. Therefore, the movements of other cars now are perceived as being even slower than they normally would be. The point is that velocity perception not only depends on the actual or immediately preceding speed of an object, but also on the object's speed over a longer time frame. We disentangled two possible factors (initial events and frequency of different types of events) that generate such under- or overestimation.

Our data demand a new model that addresses a combination of the primacy and the pure frequency effects (Figure 9). The judgment option model, proposed by Baird (1997), might be a possible candidate. He assumed that subjects merely tend to keep the rank order between stimuli as they judge them by adaptively (i.e., more or less smoothly and in the appropriate direction) changing their scale. Therefore, the principle of consistent identification (same-stimulus-same-category) is replaced by the principle of consistent ordering of the stimuli, which apparently poses fewer demands on a subject. The results of a computer simulation were found to be in a fairly good agreement with the experimental data both of Hau-

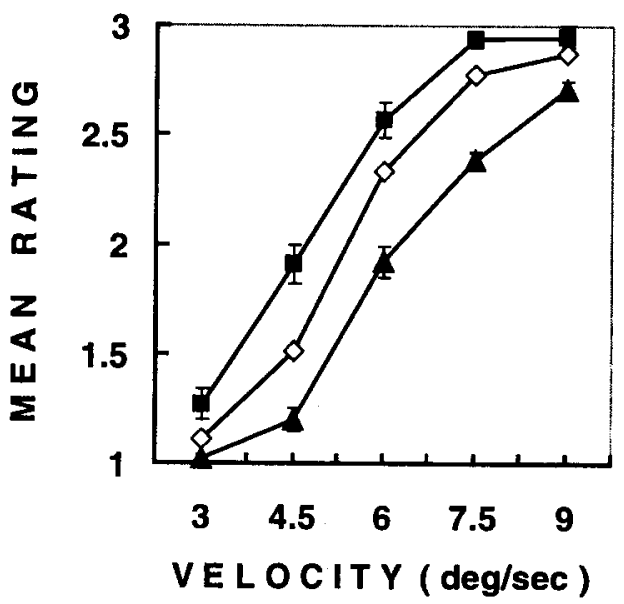

Figure 9. Mean ratings of velocity as a function of presentation order and frequency of stimuli (Experiments 2 and 3). Experimental curves for positively (squares) and for negatively (triangles) skewed sets are reproduced from Figure 4 . This conventional frequency effect represents a combination of the primacy and the pure frequency effects shown by areas under and above the intermediate curve (diamonds) that is created by using the experimental data from Figures 5 and 6. 
bensak (1992) and of Parducci and Wedell (1986). However, at present, it is not clear whether this model can deal with the transfer effects in absolute judgment. Specifically, if the subject's scale is adaptable, as is suggested in the judgment option model, why, after the context has been changed, does the scale adjustment take such a long time and still remain incomplete?

\section{REFERENCES}

Algom, D., \& Marks, L. E. (1990). Range and regression, loudness scales, and loudness processing: Toward a context-bound psychophysics. Journal of Experimental Psychology: Human Perception \& Performance, 16, 706-727.

BaIRD, J. C. (1997). Sensation and judgment: Complementarity theory of psychophysics. Mahwah, NJ: Erlbaum.

Di LOLLO, V. (1964). Contrast effects in the judgment of lifted weights. Journal of Experimental Psychology, 68, 383-387.

ERLEBaCher, A., \& SeKULER, R. (1971). Response frequency equalization: A bias model for psychophysics. Perception \& Psychophysics, 9, 315-320.

Guilford, J. P. (1954). Psychometric methods (2nd ed.). New York: McGraw-Hill.

HaubensaK, G. (1982). Eine weitere Vereinfachung der RangeFrequency-Theorie des absoluten Urteils [Another simplification of the range-frequency model for absolute judgments]. Psychologische Beiträge, 24, 352-355.

HAUBENSAK, G. (1985). Absolutes und vergleichendes Urteil: Eine Einführung in die Theorie psychischer Bezugssysteme [Absolute and comparative judgments: An introduction to the theory of psychical reference systems]. Berlin: Springer-Verlag.

HAUbenSaK, G. (1989). Primacy-Effekte in psychophysischen Urteilen [Primacy effects in psychophysical judgments]. Zeitschrift für experimentelle und angewandte Psychologie, 36, 42-56.

HAUBENSAK, G. (1992). The consistency model: A process model for absolute judgments. Journal of Experimental Psychology: Human Perception \& Performance, 18, 303-309.

Jesteadt, W., LuCE, R. D., \& Green, D. M. (1977). Sequential effects in judgments of loudness. Journal of Experimental Psychology: Human Perception \& Performance, 3, 92-104.

JOHNSON, N. L., \& KoTZ, S. (1977). Urn models and their application. New York: Wiley.

LoCKHEAD, G. R. (1984). Sequential predictors of choice in psychophysical tasks. In S. Kornblum \& J. Requin (Eds.), Preparatory states and processes (pp. 27-47). Hillsdale, NJ: Erlbaum.

Mandriota, F. J., Mintz, D. E., \& NotTerman, J. M. (1962). Visual velocity discrimination: Effects of spatial and temporal cues. Science, 138, 437-438.

MARKS, L. E. (1968). Stimulus-range, number of categories, and form of category scale. American Journal of Psychology, 81, 467-479.

PARDUCCI, A. (1954). Learning variables in the judgment of single stimuli. Journal of Experimental Psychology, 48, 24-30.

ParducCi, A. (1956). Direction of shift in the judgment of single stimuli. Journal of Experimental Psychology, 51, 169-178.

PARducci, A. (1959). An adaptation-level analysis of ordinal effects in judgment. Journal of Experimental Psychology, 58, 239-246.

Parducci, A. (1963). Range-frequency compromise in judgment. Psychological Monographs, General \& Applied, 77(Pt. 2), 1-50.
PARDuCCI, A. (1983). Category ratings and the relational character of judgment. In H.-G. Geissler \& V. Sarris (Eds.), Modern issues in perception (pp. 262-282). Berlin: VEB Deutscher Verlag der Wissenschaften.

PARDUCCI, A. (1992). Comment on Haubensak's associative theory of judgment. Journal of Experimental Psychology: Human Perception \& Performance, 18, 310-313.

Parducci, A., Calfee, R. C., Marshall, L. M., \& Davidson, L. P. (1960). Context effects in judgment: Adaptation level as a function of the mean, midpoint, and median of the stimuli. Journal of Experimental Psychology, 60, 65-77.

Parducci, A., KNobel, S., \& Thomas, C. (1976). Independent contexts for category ratings: A range-frequency analysis. Perception \& Psychophysics, 20, 360-366.

Parducci, A., \& Perrett, L. F. (1971). Category rating scales: Effects of relative spacing and frequency. Journal of Experimental Psychology Monograph, 89, 427-452.

ParducCI, A., \& Wedell, D. H. (1986). The category effect with rating scales: Number of categories, number of stimuli, and method of presentation. Journal of Experimental Psychology: Human Perception \& Performance, 12, 496-516.

Poulton, E. C. (1968). The new psychophysics: Six models for magnitude estimation. Psychological Bulletin, 69, 1-19.

Poulton, E. C. (1979). Models for biases in judging sensory magnitudes. Psychological Bulletin, 86, 777-803.

SANDER, K., \& SARrIs, V. (1997). Das "Two Stimulus-Two Choice"Paradigma für die Psychophysik: Range-Frequency-Modell und Adaptation-Level-Theorie im Vergleich [The "two stimulus-two choice" paradigm in psychophysics: A comparison of the rangefrequency model and the adaptation-level theory]. Zeitschrift für experimentelle Psychologie, 44, 431-446.

SChifferstein, H. N. J., \& FriJTERs, J. E. R. (1992). Contextual and sequential effects on judgments of sweetness intensity. Perception \& Psychophysics, 52, 243-255.

SCHÜßLER, S. (1981). Range-Frequency Theorie und Orientierungstheorie [Range-frequency theory and orientation theory]. Unpublished doctoral dissertation, Julius Maximilians University, Würzburg.

Sokolov, A. N., \& Ehrenstein, W. H. (1996). Absolute judgments of visual velocity: Frequency- or presentation-order effect? In S. C. Masin (Ed.), Fechner Day '96: Proceedings of the 12th Annual Meeting of the International Society for Psychophysics (pp. 57-62). Padua: International Society for Psychophysics.

STEVENS, S. S. (1971). Issues in psychophysical measurement. Psychological Review, 78, 426-450.

Stevens, S. S., \& Galanter, E. H. (1957). Ratio scales and category scales for a dozen perceptual continua. Journal of Experimental Psychology, 54, 377-411.

TVersKy, A., \& KaHNEMAN, D. (1974). Judgment and uncertainty. Science, 185, 1124-1130.

WARD, L. M., \& LockhEAD, G. R. (1970). Sequential effects and memory in category judgments. Journal of Experimental Psychology, 84, 27-34.

WEDELL, D. H. (1984). A process model for psychophysical judgment. Dissertation Abstracts International, 45, 3102-B. (University Microfilms International No. 8428589)

WEDELL, D. H. (1990). Methods for determining the locus of context effects in judgment. In J. P. Caverni, J. M. Fabre, \& M. Gonzalez (Eds.), Cognitive biases (pp. 285-302). New York: Elsevier.

(Manuscript received November 3, 1998; revision accepted for publication August 5, 1999.) 